\title{
PREVALENCE OF FAILED INFERIOR ALVEOLAR NERVE BLOCK (IANB) IN ACHIEVING PULPAL ANAESTHESIA IN MANDIBULAR MOLARS WITH SYMPTOMATIC IRREVERSIBLE PULPITIS
}

\author{
Mohammed Howait* and Ghadeer I. Basunbul**
}

\begin{abstract}
Introduction: Patient comfort during root canal treatment is immense for patient cooperation and endodontist efficiency. Establishing the high prevalence of failure of IANB injections in providing pulpal anaesthesia, will most likely support the investigation of supplemental techniques to ensure patient comfort.
\end{abstract}

Objectives: The study aimed to evaluate the prevalence of failed Inferior Alveolar Nerve Block (IANB) injections in achieving pulpal anaesthesia in mandibular molars diagnosed with symptomatic irreversible pulpitis when administered alone.

Methods: This study involved fifty patients, diagnosed with symptomatic irreversible pulpitis in their mandibular first and second molars. IANB injection using 2\% lidocaine with 1:100,000 epinephrine was administered to anesthetize the acutely inflamed teeth before commencing root canal treatment. Lip and tongue numbness was checked subjectively after injection to ensure successful IANB injection. Objectively, cold test was performed after 15 minutes and pain level was recorded using Visual Analogue Scale (VAS). Success was defined as no pain response by (VAS) during cavity preparation, endodontic access, or initial instrumentation.

Results: Although, majority of patient (96\%) expressed surrounding soft tissue numbness, only $73 \%$ did not feel any pain during initiating root canal treatment.

Conclusions: IANB injection alone is not enough to provide profound pulpal anaesthesia in acutely inflamed mandibular molars; therefore, supplemental injections are required to achieve patients comfort during treatment.

KEYWORDS: anaesthesia, endodontics, inferior alveolar nerve block, symptomatic irreversible pulpitis

* Assistant Professor, Department of Endodontics, King Abdulaziz University, Faculty of Dentistry, Jeddah, Saudi Arabia

** Assistant Professor, Oral and Maxillofacial Rehabilitation Department, King Abdulaziz University, Faculty of Dentistry, Jeddah, Saudi Arabia 


\section{INTRODUCTION}

Root canal treatment is widely believed to be the most painful and fearful dental procedure. ${ }^{(1,}$ 2) However, a survey conducted by the American Dental association in 2005 and 2006 stated that over 15 million elective non-surgical root canal treatment was performed in the United States each year. ${ }^{(3)}$ This high number is suggesting that dental patients appreciate the service provided by endodontists in successfully retaining teeth with pulpal or periapical disease that otherwise likely to be extracted.

Pain during endodontic procedures is an undesirable occurrence for both patients and clinicians. Pain experience in the dental office increases operator stress and anxiety while working and may lead to patient avoidance of adequate dental care and thus impair oral health and general wellbeing.(4) Local anaesthesia has proven to be effectual during performing dental procedures on a routine basis. Despite the progress in the field of pain relief and endodontics, pulpal anaesthesia of posterior mandibular teeth with the presence of irreversible pulpitis is not consistently succeeding in achieved analgesia. Higher concentration of anaesthetic solution in the pulp is usually required to ensure patient comfort. ${ }^{(5)}$ Occasionally, supplemental administration of anaesthesia is necessary despite the success of anaesthesia in the associated soft tissue and adjacent teeth, to make the dental procedure less painful. ${ }^{(6)}$

Infiltration of buccal mucosa is the main technique for anesthetizing the maxillary teeth that require dental procedures. However, this technique is not effective in the mandible as compared to the nerve block techniques due to the thickness of buccal cortical bone in the mandibular jaw. ${ }^{(7)}$ This explains the frequent use of inferior alveolar nerve block (IANB) injection technique as the main modality to anaesthetize mandibular posterior teeth. Generally, the local anaesthesia method of inferior alveolar nerve block (IANB) is the preferred technique used during performing dental procedures encompassing the mandibular teeth. ${ }^{(5)}$ Following IANB, challenges arrive in the achievement of deep pulpal anaesthesia particularly acutely inflamed mandibular molars. The proper in application of the IANB in the clinical setting demonstrates its failure in about $30 \%-45 \%$ cases. Studies has confirmed that sometimes this IAND technique alone does not produce the desired anaesthesia. Problems are likely to arise while treating mandibular teeth with irreversible pulpitis. $^{(7-9)}$

This paper intended to assess and prove that the application of IANB unaccompanied will not effectively anesthetize the pulp consistently and will not offer a solution for painless dental procedures, particularly during non-surgical root canal treatment work. Consequently, the integration of IANB and supplementary injection may simplify the treatment procedure for the practicing dentists.

\section{MATERIAL AND METHODS}

The study has been conducted by recruiting patients visiting the endodontic clinics at King Abdul Aziz University Dental Hospital, Jeddah, Saudi Arabia. Study was approved by King Abdul Aziz, Faculty of Dentistry Research Ethics Committee (REC 016-01-17) and a written consent was obtained from all patients. Healthy (ASA I, based on the American Society of Anesthesiologists (ASA) physical status classification system), 25 - 45 years old male patients were included in the study. All patients were diagnosed with symptomatic irreversible pulpitis in their first or second mandibular molars and did not consume any pain killer within the last 72 hours. Medical and dental history was recorded or updated in the clinic to gather information about the patients. Information included: demographic data, detailed chief complaint history to reach proper diagnosis.

Two endodontic residents were calibrated to administer the anaesthesia for the participating 
patients in the study. The mandibular molars were anesthetized by administrating IANB injection using 2\% lidocaine and 1:100000 epinephrine (Xylocaine with Epinephrine; Astra) before commencing the dental procedure. The cartridge of the anaesthesia was deposited slowly over a period of 60 seconds after aspiration using aspirating syringe and 25 gauge sterile dental needle. The success of IANB injection was ensured by questioning the patient about lip and tongue numbness subjectively 5 minutes after injection. Cold test was performed after 15 minutes and pain levels were recorded using Visual Analogue Scale (VAS) scale. The success of IANB injection was measured as no pain feeling by (VAS) during cavity preparations, endodontic access, or initial instrumentation.

Data was analysed with Statistical Package of Social Sciences (SPSS) software version 20.0 (SPSS Inc., Chicago, IL, USA). A simple descriptive analysis was used to define the study variables through a form of counts and percentages.

\section{RESULTS}

Although, total fifty patients were involved in this study, forty-eight patients were used for data analysis after excluding two patients because of failure to achieve subjective and objective signs of anaesthesia. A total of 50 patients were recruited from the endodontic clinic in King Abdul Aziz University Dental Hospital, all of the patients were suffering from symptomatic irreversible pulpitis (Table 1). Only 2 patients (4\%) did not report tongue and lip numbness subjectively 5 minutes after the IANB injection, whereas 48 patients $(96 \%)$ reported numbness sensation in lip and check and success of anaesthesia in adjacent teeth and soft tissue. Majority of patients (72.9\%) did not feel any pain response to cold test 15 minutes after administering the IANB injection and commencing root canal treatment. Thirteen patient $(27.1 \%)$ reported pain by VAS 15 minutes after anaesthesia administration and were recorded to represent the prevalence of IANB injection failure among mandibular molars diagnosed with symptomatic irreversible pulpitis (Table 1)

TABLE (1) Descriptive Analysis

\begin{tabular}{|l|c|c|c|}
\hline \multicolumn{1}{|c|}{ Measure } & Items & Frequency & $\begin{array}{c}\text { Percentage } \\
(\mathbf{\%})\end{array}$ \\
\hline Diagnosis & $\begin{array}{c}\text { Irreversible } \\
\text { Pulpitis }\end{array}$ & 50 & 100 \\
\hline \multirow{2}{*}{$\begin{array}{l}\text { Lip and Cheek } \\
\text { Numbness }\end{array}$} & Yes & 48 & 96 \\
\cline { 2 - 4 } & No & 2 & 4 \\
\hline \multirow{2}{*}{ Feeling of Pain } & Yes & 13 & 27.1 \\
\cline { 2 - 4 } & No & 35 & 72.9 \\
\hline
\end{tabular}

\section{DISCUSSION}

The findings of the study is consistent with the literature. Although, $96 \%$ of the participants experienced numbness in the lip, cheek, tongue and adjacent soft tissue, only $73 \%$ reported no pain feeling by (VAS) during cavity preparations, endodontic access, or initial instrumentation. Therefore, lip and soft tissue anaesthesia is not a reliable indicator for profound pulpal anaesthesia. A negative response to cold test is considered an effective tool in assessing pulpal anaesthesia. ${ }^{(10,11)}$ In addition, the administration of IANB injection alone does not guarantee a pain free intra-operative endodontic treatment for acutely inflamed mandibular posterior teeth. In the current study, the success rate of the properly administered INAB injection in anesthetizing acutely inflamed mandibular molars was $73 \%$. Different clinical studies reported a failure rate of IANB injection in anesthetizing hot posterior mandibular teeth to range between $44 \%$ and $81 \% \cdot(10,12-14)$ For treating those patients, the integration of IANB and supplementary injections using different techniques and different type of anaesthetic solutions may results in a painless dental treatment as compared to the recurrence of 
the IANB injections. ${ }^{(15-17)}$ Supplemental injections techniques include intraosseous, intra-ligamentary, intra-pulpal as well as infiltration.

Unfortunately, the use of buccal infiltration in the posterior mandibular molar region was not found to be always predictable in providing profound pulpal anaesthesia. A study speculated that following the failure of the IANB, only $58 \%$ of pulpal anaesthesia success was achieved when $4 \%$ articaine with 1:100,000 epinephrine was administered as supplemental buccal infiltration in treating mandibular posterior teeth with irreversible pulpitis. ${ }^{(18)}$ This may be as the result of the thickness of the cortical bone in the mandibular posterior region as compared to the spongy bone in the maxilla where infiltration is the main modality to anesthetize the maxillary posterior teeth. ${ }^{(7)}$

Higher anaesthesia success rate was reported during endodontic procedures performed on mandibular posterior teeth which had irreversible pulpitis when supplemental intraosseous injection using $1.8 \mathrm{ml}$ solution of either $2 \%$ lidocaine combined with 1:100,000 epinephrine or $1.5 \%$ etidocaine hydrochloride with 1:200.000 epinephrine was administered in contrast to the conventional IANB method used alone or repeating the conventional IANB method again. ${ }^{(17,19)}$ Special equipment for drilling the cortical bone and injecting the anaesthetic solution is required for adequate intraosseous injection; therefore, it is not considered the preferable modality. ${ }^{(20)}$

Studies reported that the success of intra-pulpal injection is autonomous in cases where pain is experienced upon entrance of the pulp. Intra-pulpal anaesthesia effectiveness is dependent upon the back pressure which is disseminated to the pulp through the injection of the solution in the presence of strong back pressure. The anaesthesia effect was found to be profound as well as instant with minimal systemic effect. ${ }^{(21)}$ A major drawback of this supplemental technique is the engorgement of the blood vessels in the pulp chamber which make the injection itself very painful. ${ }^{(22,23)}$

In the mandibular molar region, the combination of intra-ligamentary injection with IANB amplified the occurrence of pulpal anaesthesia for the starting twenty-three minutes. The success incidence in terms of the efficiency of the intra-ligamentary injection was observed to be $92 \%$ involving the incidence of reinjection as well. The intra-ligamentary injection technique became popular in the 1970s with the development of special dental devices dedicated for this injection technique. A 27 gauge short dental needle is recommended to deposit about $0.2 \mathrm{~mL}$ of the anesthetic solution into the gingival sulcus where the solution penetrates the fenestrations found in the alveolar socket. A strong pressure is necessary to be present when the periodontal ligament injection is given for attaining the significant rate of success in terms of anaesthesia. In case the first injection did not produce the required outcome, reinjection is often effective along the different sides of the tooth. ${ }^{(24)}$ Pain is likely to increase post-operatively after administering intra-liganmentary injections. Many subjects reported increased level of pain during the injection when compared to infiltration anaesthesia. $^{(25)}$

In conclusion, challenges arrive pertaining to the proper production of anaesthesia in mandibular posterior teeth pulp that has irreversible pulpitis despite the well documented nerve regional blocks. The results of clinical trials showed that during treating inflamed mandibular molars, it is essential to use supplemental injection because of the increased failing chances of proper anesthetization of the pulp by the application of IANB alone. To obtain profound pulpal anaesthesia and provide a pain-free dental treatment, helping both the patient and the dental practitioner, simpler options are likely to be investigated to increase the success rate of IANB injections and recommend the utilization of supplemental injection technique without collateral adverse effects. 


\section{ACKNOWLEDGEMENTS}

The authors are very thankful to all the associated personnel in any reference that contributed in/for the purpose of this research.

\section{FUNDING}

This research did not receive any specific grant from funding agencies in the public, commercial, or not-for-profit sectors.

\section{REFERENCES}

1. Udoye CI, Oginni AO, Oginni FO. Dental anxiety among patients undergoing various dental treatments in a Nigerian teaching hospital. J Contemp Dent Pract. 2005;6(2):91-8.

2. Watkins CA, Logan HL, Kirchner HL. Anticipated and experienced pain associated with endodontic therapy. The Journal of the American Dental Association. 2002; 133(1):45-54.

3. Association AD. 06 survey of dental services rendered. Chicago: American Dental Association. 2007;44.

4. Dionne RA, GORDON SM, MCCULLAGH LM, PHERO JC. Assessing the need for anesthesia and sedation in the general population. The Journal of the American Dental Association. 1998;129(2):167-73.

5. Bataineh AB, Alwarafi MA. Patient's pain perception during mandibular molar extraction with articaine: a comparison study between infiltration and inferior alveolar nerve block. Clinical oral investigations. 2016;20(8):2241-50.

6. Saberi EA, Farhadmollashahi N, Aliabadi T. A Comparison of the Anesthesia Efficacy of Articaine and Articaine plus Morphine for Buccal Infiltration in Mandibular Posterior Teeth with Irriversible Pulpitis. Zahedan Journal of Research in Medical Sciences. 2014;16(7):26-30.

7. Yadav S. Anesthetic success of supplemental infiltration in mandibular molars with irreversible pulpitis: A systematic review. Journal of conservative dentistry: JCD. 2015;18(3):182.

8. Claffey E, Reader A, Nusstein J, Beck M, Weaver J. Anesthetic efficacy of articaine for inferior alveolar nerve blocks in patients with irreversible pulpitis. Journal of endodontics. 2004;30(8):568-71.

9. Raj JD, Ramesh S. Evaluation of the anesthetic efficacy of inferior alveolar nerve blocks in dental patients-A Systematic Review. IOSR J Dent Med Sci. 2013;8:10-7.
10. Cohen HP, Cha BY, Spångberg LS. Endodontic anesthesia in mandibular molars: a clinical study. Journal of Endodontics. 1993;19(7):370-3.

11. Hsiao-Wu GW, Susarla SM, White RR. Use of the cold test as a measure of pulpal anesthesia during endodontic therapy: a randomized, blinded, placebo-controlled clinical trial. Journal of endodontics. 2007;33(4):406-10.

12. Reisman D, Reader A, Nist R, Beck M, Weaver J. Anesthetic efficacy of the supplemental intraosseous injection of $3 \%$ mepivacaine in irreversible pulpitis. Oral Surgery, Oral Medicine, Oral Pathology, Oral Radiology, and Endodontology. 1997;84(6):676-82.

13. Nusstein J, Reader A, Nist R, Beck M, Meyers WJ. Anesthetic efficacy of the supplemental intraosseous injection of $2 \%$ lidocaine with 1: 100,000 epinephrine in irreversible pulpitis. Journal of endodontics. 1998;24(7):487-91.

14. Kennedy S, Reader A, Nusstein J, Beck M, Weaver J. The significance of needle deflection in success of the inferior alveolar nerve block in patients with irreversible pulpitis. Journal of endodontics. 2003;29(10):630-3.

15. Ahmad ZH, Ravikumar H, Karale R, Preethanath R, Sukumaran A. Study of the anesthetic efficacy of inferior alveolar nerve block using articaine in irreversible pulpitis. The journal of contemporary dental practice. 2014;15(1):71-4.

16. Fan S, Chen W-1, Pan C-b, Huang Z-q, Xian M-q, Yang $\mathrm{Z}$-h, et al. Anesthetic efficacy of inferior alveolar nerve block plus buccal infiltration or periodontal ligament injections with articaine in patients with irreversible pulpitis in the mandibular first molar. Oral Surgery, Oral Medicine, Oral Pathology, Oral Radiology, and Endodontology. 2009;108(5):e89-e93.

17. Kanaa MD, Whitworth JM, Meechan JG. A prospective randomized trial of different supplementary local anesthetic techniques after failure of inferior alveolar nerve block in patients with irreversible pulpitis in mandibular teeth. Journal of Endodontics. 2012;38(4):421-5.

18. Matthews R, Drum M, Reader A, Nusstein J, Beck M. Articaine for supplemental buccal mandibular infiltration anesthesia in patients with irreversible pulpitis when the inferior alveolar nerve block fails. Journal of endodontics. 2009;35(3):343-6.

19. Webster Jr S, Drum M, Reader A, Fowler S, Nusstein J, Beck M. How effective is supplemental intraseptal anesthesia in patients with symptomatic irreversible pulpitis? Journal of endodontics. 2016;42(10):1453-7. 
20. Hargreaves KM, Keiser K. Local anesthetic failure in endodontics: mechanisms and management. Endodontic Topics. 2002;1(1):26-39.

21. Balasubramanian SK, Natanasabapathy V, Vinayachandran D. Clinical considerations of intrapulpal anesthesia in pediatric dentistry. Anesthesia, essays and researches. 2017;11(1): 1 .

22. Malamed SF. The management of pain and anxiety. Pathways of the Pulp St Louis: Mosby. $4^{\text {th }}$ Ed.1994:568-83.

23. Harris MH. The use of local anesthesia in the presence of inflammation. Oral Surgery, Oral Medicine, Oral Pathology. 1964;18(1):16-23.

24. Malamed SF. The periodontal ligament (PDL) injection: an alternative to inferior alveolar nerve block. Oral Surgery, Oral Medicine, Oral Pathology. 1982;53(2):117-21.

25. Al-Shayyab MH. Periodontal ligament injection versus routine local infiltration for nonsurgical single posterior maxillary permanent tooth extraction: comparative double-blinded randomized clinical study. Therapeutics and clinical risk management. 2017;13:1323. 